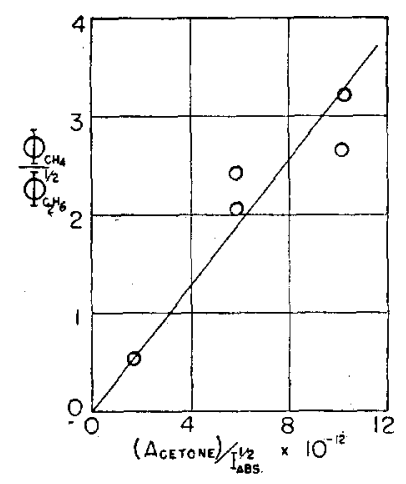

FIG. 4. A plot of Eq. (3) at $122^{\circ} \mathrm{C}$.

acetoné :

$\mathrm{CH}_{2} \mathrm{COCH}_{3}+\mathrm{CH}_{2} \mathrm{COCH}_{3}$

$$
=\left(\mathrm{CH}_{2} \mathrm{COCH}_{3}\right)_{2} ; k_{4}\left(\mathrm{CH}_{2} \mathrm{COCH}_{3}\right)^{2} \text {. }
$$

This compound has been identified by Rice,
Rodowskas, and Lewis ${ }^{12}$ as a product in the reaction of methyl radicals with acetone at $350^{\circ} \mathrm{C}$.

The slope of the straight line in Fig. 3 gives $k_{2} / k^{\frac{1}{2}}=2.70 \times 10^{-14}$ at $26^{\circ} \mathrm{C}$. Figure 4 gives $k_{2} / k_{1}{ }^{\frac{1}{2}}$ $=3.19 \times 10^{-13}$ at $122^{\circ} \mathrm{C}$. Writing $k_{2}=a_{2} e^{-E_{2} / R T}$ and $k_{1}=a_{1} e^{-E_{1} / R T}$, these values may be used to calculate $E_{2}-\frac{1}{2} E_{1}=5700 \mathrm{cal}$. per mole. The $T^{1}$ factor in the pre-exponential term has been taken into account in this calculation. Taking the value of Cunningham and Taylor $^{13}$ for reaction (1), $E_{1}<1.5 \mathrm{kcal} . /$ mole, gives

$$
E_{2}=6500 \text { calories per mole. }
$$

This value is only slightly lower than the value of $7.1 \mathrm{kcal}$. mole obtained by Allen. ${ }^{11}$

${ }^{12}$ F. O. Rice, E. L. Rodowskas, and W. R. Lewis, J. Am. Chem. Soc. 56, 2497 (1934).

${ }_{13} \mathrm{~J}$. P. Cunningham, and H. S. Taylor, J. Chem. Phys. 6, 359 (1938).

\title{
Thermodynamic Properties of the Internal Rotator. Double Minimum with Attractive Forces
}

\author{
J. O. HALFORD \\ Department of Chemistry, University of Michigan, Ann Arbor, Michigan
}

(Received March 22, 1948)

\begin{abstract}
A previously outlined general method of calculating thermodynamic properties for an unsymmetrical internal rotation is applied to a double minimum with attractive forces, for which the potential energy has two maxima at the same height and two minima at different heights. The entropy is lower than that of the system of two equal potential valleys or that for a single valley, and can be lower than the former by as much as $R \ln 2$ when the potential barriers are high. This decrease tends to disappear in the region of close approach to free rotation, when the barriers are lower than $R T$. For very high potential barriers, if one of two equal minima is raised, the system acquires the properties of a simple harmonic oscillator.
\end{abstract}

$I^{\mathrm{N}}$ $\mathrm{N}$ a recent paper ${ }^{1}$ it was shown that graphical old quaritum-theory action integrals could be used to obtain mean positions of the energy levels of the symmetrical internal rotator from which the contributions of the internal rotation to the thermodynamic properties could be closely approximated. More recently a scheme has been proposed for applying the old quantum theory when the rotation is not symmetrical. By assuming for the potential energy function a

1 J. O. Halford, J. Chem. Phys. 15, 645 (1947).

2 J. O. Halford, J. Chem. Phys. 16, 410 (1948). succession of linked sections of simple cosine curve, it is possible to use the graphical integrations carried out for the symmetrical rotator to evaluate the properties of the unsymmetrical system. The results of these integrations are contained in a table (Table II, reference 2) showing $\rho$, a quantum-number index, as a function of $E / V$, the ratio of the energy to the barrier height when the zero of energies is placed at the bottom of the potential valley. For a rotation or vibration through the region above one or more potential minima, a new quantum. 
number index $R$ is given by the equation

$$
\mathcal{R}=\sum\left(\rho_{i} / a_{i}\right)\left(V_{i} / V_{0}\right)^{\frac{1}{2}} / 2=r /\left[Q_{f}\left(V_{0} / R T\right)^{\frac{1}{2}}\right]
$$

in which $r$ is the quantum number, $Q_{f}$ is the limiting partition function for free rotation, $\rho_{i}$ is taken from the table at the selected value of $E_{i} / V_{i}$ at which $\Omega$ is sought, $a_{i}=a_{0} V_{0} / V_{i}$, and $\sum 1 / a_{i}=2$ for the complete rotational cycle. The summation includes two terms for each potential valley in which, or above which, the vibration or rotation occurs. A relation between $R$ and $E / V_{0}$ is then set up, which by Eq. (1) permits the selection of ratios $E / V_{0}$ for any assigned set of quantum numbers $r$.

The establishment of the quantum-number index as a function of $E / V_{0}$ by the old quantum theory can be done in only one way for a given potential energy function. There is some latitude of choice, however, in the selection of quantum numbers for the representative mean positions of the energy levels, and this latitude will lead to uncertainties in the thermodynamic properties under certain limiting conditions. In calculations with the symmetrical rotator, it is possible to find the rules of assignment which will reproduce properties calculated by more rigorous methods. ${ }^{1,3}$ When the rotator is unsymmetrical, however, there is no more rigorous solution available for comparison, and it becomes necessary to assume that an assignment of quantum numbers consistent with experience acquired with the symmetrical rotator will yield accurate results. When this experience cannot be applied unequivocally, the positions of some of the states will be in doubt.

For this reason, and because the method is an approximation based upon a postulate of limited validity, there will be regions of the variables $V_{i}$, $T$, and $Q_{f}$, in which accurate thermodynamic properties cannot be calculated. This is true to some degree of any method of evaluating these properties, and the practical question for any one method is whether molecules exist that have properties falling outside the region of accurate calculation.

The limitations of the proposed approximation are not obvious from superficial examination, but they should become apparent as the attempt

\footnotetext{
${ }^{3}$ K. S. Pitzer and W. D. Gwinn, J. Chem. Phys, 10, 428 (1942).
}

to apply the method is made. The chosen approach to an understanding of these limits has been first, to find the conditions necessary for the accurate calculation of the known properties of the symmetrical rotator, second, to apply the general method for the unsymmetrical rotator to a double minimum with repulsive forces for which two unequal potential maxima occur between minima at the same level, and third, to study the double minimum with attractive forces where the maxima are at the same height but one minimum lies above the other. The first two of these studies appear in earlier publications. The third is the basis for the present paper.

The conditions sufficient to reproduce the $n$-fold symmetrical rotator properties are:

(1) Each $n$th rotational pair, as identified by the quantum numbers of the limiting free rotator, is counted.

(2) Quantization is performed over the entire rotational cycle and half the vibrational cycle. The vibrational states are placed at the positions $r \pm \frac{1}{4}$, the rotational states at $r \pm V / 4 E$, where $r$ is an integer, $V$ is the barrier height, and $E$ is the energy at the mean position $r$.

(3) When a pair of states is split across the potential maximum, the upper component is so placed that the mean quantum number is an integer.

(4) When fewer than three states lie below the maximum, they are adjusted downward empirically to keep the partition function below its limiting value for free rotation.

With suitable modifications, but without essential changes, these conditions are applicable to the double minimum with repulsive forces:

(1) All states as identified by their quantum numbers in the free rotation limit are counted.

(2) For quantization over the whole rotational cycle and half the vibrational cycles, the states are placed at $r \pm \frac{1}{4}$ below the lower maximum, at $r \pm\left(\frac{1}{4}+V_{2} / 4 E\right)$ between the maxima and at $r \pm\left(V_{1}+V_{2}\right) / 4 E$ in the rotational region. Here $V_{1}$ is the higher barrier. Below the lower maximum the states have double weight.

(3) The average position of the pairs is again maintained at integral values of the quantum number.

(4) When fewer than three vibrational states occur within a potential valley, below the lower 
TABLE I. Thermodynamic properties of the double minimum internal rotator with attractive forces (cal./mole/degree).

\begin{tabular}{|c|c|c|c|c|c|c|c|c|c|c|c|c|c|c|c|c|}
\hline & & $1 / Q_{f}$ & 0.05 & & & 0.10 & & & 0.20 & & & 0.30 & & & 0.40 & \\
\hline$R T$ & & i $\backslash-F / T$ & $H / T$ & $S$ & $-F / T$ & $H / T$ & $S$ & $-F / T$ & $\dot{H} / T$ & $S$ & $-F / T$ & $H / T$ & $s$ & $-F / T$ & $H / T$ & $s$ \\
\hline 1 & $\begin{array}{l}1 \\
\frac{3}{4} \\
\frac{1}{2} \\
\frac{1}{4} \\
0\end{array}$ & $\begin{array}{l}5.25 \\
5.11 \\
5.03 \\
5.04 \\
5.17\end{array}$ & $\begin{array}{l}1.58 \\
1.74 \\
1.80 \\
1.78 \\
1.66\end{array}$ & $\begin{array}{l}6.83 \\
6.84 \\
6.83 \\
6.82 \\
6.83\end{array}$ & $\begin{array}{l}4.03 \\
3.88 \\
3.78 \\
3.77 \\
3.88\end{array}$ & $\begin{array}{l}1.42 \\
1.59 \\
1.68 \\
1.67 \\
1.58\end{array}$ & $\begin{array}{l}5.45 \\
5.47 \\
5.46 \\
5.44 \\
5.45\end{array}$ & & $X$ & & & $X$ & & & $X$ & \\
\hline 4 & $\begin{array}{l}1 \\
\frac{3}{4} \\
\frac{1}{2} \\
\frac{1}{1} \\
0\end{array}$ & $\begin{array}{l}3.96 \\
3.36 \\
3.26 \\
3.44 \\
3.79\end{array}$ & $\begin{array}{l}1.86 \\
2.40 \\
2.30 \\
2.12 \\
2.02\end{array}$ & $\begin{array}{l}5.82 \\
5.76 \\
5.57 \\
5.56 \\
5.81\end{array}$ & $\begin{array}{l}2.90 \\
2.27 \\
2.06 \\
2.26 \\
2.58\end{array}$ & $\begin{array}{l}1.58 \\
2.11 \\
2.11 \\
1.93 \\
1.86\end{array}$ & $\begin{array}{l}4.48 \\
4.38 \\
4.17 \\
4.19 \\
4.44\end{array}$ & $\begin{array}{l}2.09 \\
1.41 \\
1.20 \\
1.27 \\
1.51\end{array}$ & $\begin{array}{l}1.12 \\
1.72 \\
1.69 \\
1.58 \\
1.58\end{array}$ & $\begin{array}{l}3.21 \\
3.14 \\
2.89 \\
2.85 \\
3.09\end{array}$ & & $X$ & & & $X$ & \\
\hline 9 & $\begin{array}{l}1 \\
\frac{3}{4} \\
\frac{1}{2} \\
\frac{1}{4} \\
0\end{array}$ & $\begin{array}{l}3.20 \\
2.20 \\
2.32 \\
2.58 \\
2.96\end{array}$ & $\begin{array}{l}1.58 \\
2.03 \\
1.79 \\
1.66 \\
1.81\end{array}$ & $\begin{array}{l}4.78 \\
4.22 \\
4.11 \\
4.24 \\
4.77\end{array}$ & $\begin{array}{l}2.28 \\
1.22 \\
1.27 \\
1.50 \\
1.82\end{array}$ & $\begin{array}{l}1.20 \\
1.67 \\
1.44 \\
1.48 \\
1.58\end{array}$ & $\begin{array}{l}3.47 \\
2.89 \\
2.71 \\
2.98 \\
3.41\end{array}$ & $\begin{array}{l}1.68 \\
0.55 \\
0.52 \\
0.66 \\
0.90\end{array}$ & $\begin{array}{l}0.66 \\
1.15 \\
0.96 \\
1.04 \\
1.20\end{array}$ & $\begin{array}{l}2.34 \\
1.70 \\
1.48 \\
1.70 \\
2.10\end{array}$ & $\begin{array}{l}1.50 \\
0.34 \\
0.25 \\
0.34 \\
0.50\end{array}$ & $\begin{array}{l}0.36 \\
0.84 \\
0.63 \\
0.72 \\
0.89\end{array}$ & $\begin{array}{l}1.86 \\
1.18 \\
0.88 \\
1.06 \\
1.40\end{array}$ & & $X$ & \\
\hline 16 & $\begin{array}{l}1 \\
\frac{3}{4} \\
\frac{1}{2} \\
\frac{1}{4} \\
0\end{array}$ & $\begin{array}{l}2.76 \\
1.59 \\
1.82 \\
2.09 \\
2.44\end{array}$ & $\begin{array}{l}1.40 \\
1.57 \\
1.53 \\
1.60 \\
1.69\end{array}$ & $\begin{array}{l}4.16 \\
3.16 \\
3.54 \\
3.69 \\
4.13\end{array}$ & $\begin{array}{l}1.96 \\
0.70 \\
0.89 \\
1.09 \\
1.38\end{array}$ & $\begin{array}{l}0.94 \\
1.18 \\
1.15 \\
1.27 \\
1.41\end{array}$ & $\begin{array}{l}2.90 \\
1.87 \\
2.09 \\
2.36 \\
2.79\end{array}$ & $\begin{array}{l}1.52 \\
0.22 \\
0.28 \\
0.40 \\
0.58\end{array}$ & $\begin{array}{l}0.39 \\
0.61 \\
0.62 \\
0.76 \\
0.94\end{array}$ & $\begin{array}{l}1.91 \\
0.83 \\
0.99 \\
1.16 \\
1.52\end{array}$ & & $X$ & & $\begin{array}{l}1.39 \\
0.05 \\
0.04 \\
0.07 \\
0.14\end{array}$ & $\begin{array}{l}0.06 \\
0.22 \\
0.15 \\
0.24 \\
0.39\end{array}$ & $\begin{array}{l}1.45 \\
0.26 \\
0.19 \\
0.32 \\
0.53\end{array}$ \\
\hline
\end{tabular}

maximum, they are adjusted downward empirically by means of the same equation that was used for the symmetrical rotator. The adjustment is made separately, however, for each of the two unequal sides of the valley, and the mean of the two adjustments so obtained is applied.

For the double minimum with attractive forces it is no longer possible to meet all these conditions clearly:

(1) The number of states counted, in relation to the free rotator, is the same as for the double minimum with repulsive forces.

(2) This condition is met in the same manner as before.

(3) If the number of states below the potential maxima is even, the lowest rotational state will be the upper component of a pair, but there is no way to identify one of the vibrational states as the lower component. The scale of quantum numbers for the rotational region, however, can be projected below the potential maxima, and, if the rotational component is set at $r+\frac{1}{4}$, the location will be consistent with that used in the two cases previously considered. The effect of errors made by considerable shifts in the position of this state is negligible in all of the calculations summarized below.

(4) Downward adjustment for fewer than three states in the more shallow valley cannot be made by the empirical equation heretofore used because this equation would place the last state, as the lesser potential barrier approached zero, incorrectly at exactly the position of the potential maxima. It is found, however, within the limits of Table I that the adjustment can be neglected without introducing serious errors. The energies of the states in question are such that they may be lowered, by amounts larger than the empirical corrections previously made, without significant effects upon the thermodynamic properties.

It works out, then, that some doubt exists about the representative mean positions of the energy states in shallow valleys or immediately above the potential maxima. A limitation is thus placed upon the accurate application of the proposed approximate method for calculating the thermodynamic properties of the general unsymmetrical internal rotator, but from the cases thus far considered it appears that errors from these sources will not seriously interfere, in general, with the use of the method. At least there should be many potential energy functions for real molecules which will permit accurate evaluations by the scheme under consideration.

As a first step in resolving the questions just raised it would be useful to have a way to identify 
each state of the hindered rotator with an individual level of the limiting free rotator system. Such an identification appears to have meaning because below any energy for which the effect of the hindering potential is negligible, the number of states is independent of the detail of the potential energy curve. If the restricting potential is slowly diminished, whether the relative positions of the maxima are maintained or not, the levels will rise in the valleys and escape into the rotational region, finally fitting themselves into the free rotator system of states. Presumably, then, each level of a restricted rotator could be labeled according to the place it would take in free rotation if the barrier were removed.

The identification can be made for the symmetrical rotator or for the unsymmetrical rotator with equivalent valleys that are identical or reflecting in character. When the two valleys are different, however, the labeling of the states is not clearly indicated. If the barrier system is diminished to zero without changing its shape, the levels will retain, at least approximately, their relative positions on the scale of energies. If, however, one barrier is first removed by raising a minimum, the levels in one valley will be poured into the neighboring valley in inverse order, and, when the second barrier is subsequently diminished, will retain this new order in to the free rotation limit.

It appears, then, that there is no way to determine what limit is approached in the single minimum system by a state in the secondary valley as this valley is eliminated by raising its minimum. Likewise, for a rotational doublet divided across the potential maximum, it is not clear which vibrational state is a component of the pair. By trial, the location $r-\frac{1}{4}$, which follows from the position of the upper component at $r+\frac{1}{4}$, is a weighted mean of the positions of the uppermost states in the two valleys.

For the double minimum with attractive forces, calculations of the free energy $(-F / T)$, the heat content $(H / T)$, and the entropy $(S)$ have been made for five barrier ratios $\left(V_{2} / V_{1}\right)$ at each of several representative combinations of the variables $1 / Q_{f}$ and $V_{1} / R T$. The results are summarized in Table $I$. When the barriers are equal, the properties are taken from the tables of Pitzer and Gwinn at $2 / Q_{f}, V / R T$, and the entropy and free energy $(-F / T)$ are increased by $R \ln 2$, with $R$ taken to be 1.9869 . For $V_{2}=0$, the single minimum limit, the values are taken directly from the tables at $1 / Q_{f}, V / R T$. For each of the remaining three barrier ratios the relation between $R$ and $E / V_{1}$ has been set up and interpreted according to Eq. (1) to obtain the permitted values of the ratio $E / V_{1}$ for use in determining the thermodynamic properties. In this connection, the energy ratios for the two valleys are related according to the equation

$$
E_{2} / V_{2}=1-\left(V_{1} / V_{2}\right)\left(1-E_{1} / V_{1}\right) .
$$

When $V_{1}=R T$ the entropy is for practical purposes independent of the barrier ratio $V_{2} / V_{1}$. At higher values of $V_{1} / R T$, however, the entropy decreases as the barrier ratio is lowered by raising one of two equal minima, and the property passes through a minimum before the second valley disappears at $V_{2} / V_{1}=0$. The maximum entropy decrease becomes more pronounced as $1 / Q_{f}$ or $V_{1} / R T$ is increased, and would, in the limit, reach $R \ln 2$, or 1.38 for high values of $V_{1} / R T$ for which the populations of excited states had become negligible. It appears that for real systems with $V_{1} / R T$ of the order of 5 , the entropy might be lower by as much as 0.5 cal./mole/degree than that for two equal potential valleys.

We may go continuously from the case of equal valleys to the single minimum limit either by lowering a maximum, or, as in the problem now under consideration, by raising a minimum. In the former case the entropy passes through a maximum, in the latter through a minimum. The extreme range of entropy values for the double minimum, with both attractive and repulsive forces considered, is of the order of 1.5 entropy units and approaches $500 \mathrm{cal}$. on the energy scale at room temperature.

Again, as in the system with repulsive forces, the heat content passes through a maximum and a minimum with changing barrier ratio, but the minimum tends to disappear at the lower values of $V_{1} / R T$.

It is of some practical interest that at $V_{1}=R T$ the entropy remains invariant between the limits 
of the single minimum and the equal double minimum system. This is true for the intermediate situations produced either by lowering a maximum or raising a minimum. In other words, if the conditions are such that the entropy is the same in the limits, it will not change appreciably during the transition from one limit to the other by either of the two routes thus far considered. It is probably true that at still lower values of $V_{1} / R T$, with $V_{1}$ the higher potential barrier, the entropy will remain the same for all variations of the double minimum system produced by raising a minimum or lowering a maximum.

This conclusion will certainly be valid for all three thermodynamic properties in the region of close approach to free rotation, where for a given moment of inertia the differences between symmetrical systems are determined solely by the symmetry number. It must also be true that the invariant character will extend to higher values of $V_{1} / R T$ for the entropy than for the free energy or heat content per degree. At low potential barriers the properties are largely determined by the location of states having energies less than $R T$, and for such states a disturbance of position tends to affect the free energy and heat content in opposite directions. Consequently, an increase in the barrier height which disturbs mainly states having energies less than
$R T$ will produce relatively little change in the entropy: Although there appears to be no rigorous method of demonstrating how far up the $V_{1} / R T$ scale the invariance will go, it seems safe to assume that below any given level the constancy will improve. The invariance found at $V_{1}=R T$ can therefore be assumed to hold for $V_{1}<R T$ without much danger of error. For the double minimum, for $V_{1} \ll R T$, the entropy deficiency below free rotation is thus taken to be independent of the barrier ratio, and may be obtained from the tables for the symmetrical rotator as determined by the higher of the potential barriers. It becomes unnecessary to use the method outlined here in the region of lowest values of $V_{1} / R T$, where uncertainties in the representative location of states could lead to inaccurate results.

In the region of high potential barriers relative to $R T$, the system acts like two harmonic oscillators at equilibrium, having a number of states placed at higher levels than their positions in either the single or the equal double minimum limit. This tends to decrease both the summations which enter into the three thermodynamic properties and will cause all of these properties to pass through minima between the two limits. This tendency persists, in the free energy $(-F / T)$ and the entropy, into regions far removed from the harmonic oscillator limit. 